\title{
Global solutions of approximation problems in Hilbert spaces
}

\author{
Maximiliano Contino ${ }^{\mathrm{a}, \mathrm{b}, *}$, María Eugenia Di Iorio y Lucero $^{\mathrm{b}}$, Guillermina Fongi ${ }^{\mathrm{c}}$ \\ ${ }^{a}$ Facultad de Ingeniería, Universidad de Buenos Aires \\ Paseo Colón 850 \\ (1063) Buenos Aires, Argentina \\ "Instituto Argentino de Matemática "Alberto P. Calderón" \\ CONICET \\ Saavedra 15, Piso 3 \\ (1083) Buenos Aires, Argentina \\ ${ }^{c}$ Centro Franco Argentino de Ciencias de la Información y de Sistemas \\ CONICET \\ Ocampo y Esmeralda \\ (2000) Rosario, Argentina
}

\begin{abstract}
We study three well-known minimization problems in Hilbert spaces: the weighted least squares problem and the related problems of abstract splines and smoothing. In each case we analyze the solvability of the problem for every point of the Hilbert space in the corresponding data set, the existence of an operator that maps each data point to its solution in a linear and continuous way and the solvability of the associated operator problem in a fixed $p$-Schatten norm.
\end{abstract}

Keywords: Abstract spline problems, Schatten $p$ lasses, optimal inverses 2000 MSC: 47A05, 46C05, 41A65

\section{Introduction}

In this work we focus our attention on the following well-known approximation and interpolation problems: the weighted least squares problem and the related problems of abstract splines and smoothing. Weighted least squares problems were studied in [12] and some applications include Sard's approximation, least squares and curve fitting processes on a closed subspace [25], signal processing [16, 17] and sampling theory [3, 32]. Regarding the abstract spline and smoothing problems, Atteia [5] obtained an abstract formulation which resumed most of the spline-type functions. This theory was developed by many authors, see for example Anselone and Laurent [4], Shekhtman [30], de Boor [6], Izumino [20] and the surveys by Champion, Lenard and Mills [7, 8]. The abstract spline and smoothing problems were generalized to bounded linear operators in 14] and have been applied in many areas, such as approximation theory, numerical analysis and statistics, among others. See, for example [28], 29] and [19].

We study the conditions under which these minimization problems admit a bounded global solution, i.e., when it is possible to guarantee not only the existence of solutions for every point (of a Hilbert space) but also the existence of an operator that assigns to each point a solution in a linear and continuous way. We also study operator versions of these problems when the $p$-Schatten norms are considered and we relate the existence of solutions of such problems to the existence of bounded global solutions. Let us fix some notations: $\mathcal{H}, \mathcal{E}, \mathcal{F}$ are separable complex Hilbert spaces, $L(\mathcal{H}, \mathcal{F})$ is the set of bounded linear operators from $\mathcal{H}$ to $\mathcal{F}$ and $L(\mathcal{H}):=L(\mathcal{H}, \mathcal{H})$.

\footnotetext{
* Corresponding author

Email addresses: mcontino@fi.uba.ar (Maximiliano Contino), eugenia.diiorio@gmail.com (María Eugenia Di Iorio y Lucero), fongi@cifasis-conicet.gov.ar (Guillermina Fongi)
} 
The weighted least squares problem

Given $A \in L(\mathcal{H}, \mathcal{F}), W \in L(\mathcal{F})$ positive (semidefinite) and $x \in \mathcal{F}$, a weighted least squares solution (or $W$-LSS) of the equation $A z=x$ is a vector $u \in \mathcal{H}$ such that

$$
\|A u-x\|_{W} \leq\|A z-x\|_{W}, \quad \text { for every } z \in \mathcal{H},
$$

where $\|x\|_{W}=\langle W x, x\rangle$ is the seminorm associated to $W$.

Our main concern is to determine conditions for the existence of global solutions of WLSP). First, we analyze the existence of solutions of (WLSP for every $x \in \mathcal{F}$. Also, we study the existence of a bounded global solution of WLSP or a $W$-inverse of $A$, i.e., when there exists an operator $G \in L(\mathcal{F}, \mathcal{H})$ such that, for each $x \in \mathcal{F}, G x$ is a $W$-LSS of $A z=x$, see [26, 9]. Finally, we study the following operator least squares problem: given $A \in L(\mathcal{H}, \mathcal{F})$ (not necessarily with closed range) and $W \in L(\mathcal{F}$ ) positive such that $W^{1 / 2} \in S_{p}$ for some $1 \leq p<\infty$, analyze the existence of

$$
\min _{X \in L(\mathcal{F}, \mathcal{H})}\|A X-I\|_{p, W}
$$

where $\|Y\|_{p, W}=\left\|W^{1 / 2} Y\right\|_{p}$, for $Y \in L(\mathcal{F})$. Problem (OWLSP) was studied in 11] when $A \in L(\mathcal{H})$ is a closed range operator.

\section{Spline and smoothing problems}

A similar approach as in the case of the weighted least squares problem will be done for the spline and the smoothing problems. Consider $T \in L(\mathcal{H}, \mathcal{E})$ and $V \in L(\mathcal{H}, \mathcal{F})$.

The classical spline problem: given $f_{0} \in R(V)$, determine whether there exists

$$
\min \|T h\| \text {, subject to } V h=f_{0} .
$$

The classical smoothing problem: given $f_{0} \in \mathcal{F}$, analyze if there exists

$$
\min _{h \in \mathcal{H}}\left(\|T h\|^{2}+\left\|V h-f_{0}\right\|^{2}\right) .
$$

For each of the above two problems we study the existence of different global solutions as in the case of the weighted least squares problem.

In conclusion, for each of the three problems presented, we give conditions for the existence of solutions for every point in the corresponding data set, the existence of an operator that provides the solution at every data point in a continous way and the solution of the operator version of each of these problems considering the $p$-Schatten norms. We compare the different approaches and establish necessary and sufficient conditions for the existence of the described global solutions.

The paper is organized as follows. In Section 2 we recall the notion of compatibility between a positive operator and a closed subspace of $\mathcal{F}$. Also, we collect certain properties of the Schatten class operators that will be used along the paper. In Section 3, we study global solutions of the weighted least squares problem (WLSP), where the range of $A$ is not necessarily closed. It is proved that $A$ admits a $W$-inverse if and only if $R(A)+N(W)$ is closed and the pair $(W, R(A)+N(W))$ is compatible. This generalizes the fact that the classical least squares problem $\min _{z \in \mathcal{H}}\|A z-x\|$ admits a solution for every $x \in \mathcal{F}$ if and only if $R(A)$ is closed. Also, we show that (WLSP) admits a solution for every $x \in \mathcal{F}$ if and only if $A$ admits a $W$-inverse, or equivalently, if (OWLSP) admits a solution.

In Section 4, conditions for the existence of bounded global solutions in both the spline and the smoothing problems are given. The solutions of these problems are compared to the solutions of the corresponding operator problems in the $p$-Schatten class. Finally, $W$-optimal inverses are compared to $W$-inverses and another connection between (WLSP) and the smoothing problem is given.

\section{Preliminaries}

Throughout $\mathcal{H}, \mathcal{E}, \mathcal{F}$ are separable complex Hilbert spaces, $L(\mathcal{H}, \mathcal{F})$ is the set of bounded linear operators from $\mathcal{H}$ to $\mathcal{F}, L(\mathcal{H}):=L(\mathcal{H}, \mathcal{H})$, and $L(\mathcal{H})^{+}$denotes the cone of semidefinite positive operators. 
The symbol $\leq$ stands for the order in $L(\mathcal{H})$ induced by $L(\mathcal{H})^{+}$, i.e., given $A, B \in L(\mathcal{H}), A \leq B$ if $B-A \in L(\mathcal{H})^{+}$. For any $A \in L(\mathcal{H}, \mathcal{F})$, its range and nullspace are denoted by $R(A)$ and $N(A)$, respectively. Finally, $A^{\dagger}$ denotes the Moore-Penrose inverse of the operator $A \in L(\mathcal{H}, \mathcal{F})$.

Given two closed subspaces $\mathcal{M}$ and $\mathcal{N}$ of $\mathcal{H}, \mathcal{M} \dot{+} \mathcal{N}$ denotes the direct sum of $\mathcal{M}$ and $\mathcal{N}$. Moreover, $\mathcal{M} \oplus \mathcal{N}$ stands for their (direct) orthogonal sum and $\mathcal{M} \ominus \mathcal{N}:=\mathcal{M} \cap(\mathcal{M} \cap \mathcal{N})^{\perp}$.

Consider also, the classical inner product on $\mathcal{E} \oplus \mathcal{H}$

$$
\left\langle(e, h),\left(e^{\prime}, h^{\prime}\right)\right\rangle=\left\langle e, e^{\prime}\right\rangle+\left\langle h, h^{\prime}\right\rangle, \text { for } e, e^{\prime} \in \mathcal{E}, h, h^{\prime} \in \mathcal{H},
$$

together with the associated norm $\|(e, h)\|^{2}=\langle e, e\rangle+\langle h, h\rangle$, for $(e, h) \in \mathcal{E} \oplus \mathcal{H}$.

If $\mathcal{H}$ is decomposed as a direct sum of closed subspaces $\mathcal{H}=\mathcal{M} \dot{+} \mathcal{N}$, the projection onto $\mathcal{M}$ with nullspace $\mathcal{N}$ is denoted by $P_{\mathcal{M} / \mathcal{N}}$ and $P_{\mathcal{M}}=P_{\mathcal{M} / \mathcal{M}^{\perp}}$. Also, $\mathcal{Q}$ denotes the subset of $L(\mathcal{H})$ of oblique projections, i.e. $\mathcal{Q}=\left\{Q \in L(\mathcal{H}): Q^{2}=Q\right\}$.

Given $W \in L(\mathcal{F})^{+}$and a (non necessarily closed) subspace $\mathcal{S}$ of $\mathcal{F}$, the $W$-orthogonal complement of $\mathcal{S}$ is $\mathcal{S}^{\perp_{W}}=\{x \in \mathcal{F}:\langle W x, y\rangle=0, y \in \mathcal{S}\}=W^{-1}\left(\mathcal{S}^{\perp}\right)=W(\mathcal{S})^{\perp}$. If $\mathcal{S}$ is a closed subspace of $\mathcal{F}$, the pair $(W, \mathcal{S})$ is compatible if there exists $Q \in \mathcal{Q}$ with $R(Q)=\mathcal{S}$ such that $W Q=Q^{*} W$.

The next proposition, proved in [13, Prop. 3.3], characterizes the compatibility of the pair $(W, \mathcal{S})$.

Proposition 2.1. Consider $W \in L(\mathcal{F})^{+}$and a closed subspace $\mathcal{S} \subseteq \mathcal{F}$. Then the pair $(W, \mathcal{S})$ is compatible if and only if $\mathcal{F}=\mathcal{S}+\mathcal{S}^{\perp_{W}}$.

The notion of Schur complement of $W$ to $\mathcal{S}$, for an operator $W \in L(\mathcal{F})^{+}$and a closed subspace $\mathcal{S} \subseteq \mathcal{F}$, was introduced by M. G. Krein in 22] and later rediscovered by Anderson and Trapp [2]. They proved that the set $\left\{X \in L(\mathcal{F}): 0 \leq X \leq W\right.$ and $\left.R(X) \subseteq \mathcal{S}^{\perp}\right\}$ has a maximum element. The Schur complement of $W$ to $\mathcal{S}$ is defined by

$$
W_{/ \mathcal{S}}:=\max \left\{X \in L(\mathcal{F}): 0 \leq X \leq W \text { and } R(X) \subseteq \mathcal{S}^{\perp}\right\} .
$$

Let $T \in L(\mathcal{H}, \mathcal{E})$ be a compact operator. By $\left\{\lambda_{k}(T)\right\}_{k \geq 1}$ we denote the eigenvalues of $|T|:=$ $\left(T^{*} T\right)^{1 / 2} \in L(\mathcal{H})$, where each eigenvalue is repeated according to its multiplicity. Let $1 \leq p<\infty$, we say that $T$ belongs to the $p$-Schatten class $S_{p}(\mathcal{H}, \mathcal{E})$, if $\sum_{k \geq 1} \lambda_{k}(T)^{p}<\infty$ and, the $p$-Schatten norm is given by $\|T\|_{p}:=\left(\sum_{k>1} \lambda_{k}(T)^{p}\right)^{1 / p}$. For short, we write $S_{p}:=S_{p}(\mathcal{H}, \mathcal{H})$. The set $S_{p}(\mathcal{H}, \mathcal{E})$ is a vector space and $T \in S_{p}(\mathcal{H}, \mathcal{E})$ if and only if $|T| \in S_{p}$ (see [33, Theorem 7.6 and Theorem 7.8]). If $T \in S_{p}(\mathcal{H}, \mathcal{E})$, then

$$
\|T\|_{p}^{p}=\operatorname{tr}\left(|T|^{p}\right)
$$

where tr denotes the trace of an operator.

Observe that, $|T| X \in S_{p}$ for every $X \in L(\mathcal{H})$ and $\|T X\|_{p}=\||T| X\|_{p}$. The reader is referred to [27, 31, 33] for further details on these topics.

The following result will be useful along this paper, for its proof see [11, Proposition 2.9]. A more general result can be found in [21, Proposition 2.5].

Proposition 2.2. Let $S, T \in S_{p}$ for some $1 \leq p<\infty$. If $T^{*} T \leq S^{*} S$ then $\|T\|_{p} \leq\|S\|_{p}$.

The Fréchet derivative will be instrumental to prove some results. We recall that, for a Banach space $(\mathcal{E},\|\cdot\|)$ and an open set $\mathcal{U} \subseteq \mathcal{E}$, a function $F: \mathcal{E} \rightarrow \mathbb{R}$ is said to be Fréchet differentiable at $X_{0} \in \mathcal{U}$ if there exists $D F\left(X_{0}\right)$ a bounded linear functional such that

$$
\lim _{Y \rightarrow 0} \frac{\left|F\left(X_{0}+Y\right)-F\left(X_{0}\right)-D F\left(X_{0}\right)(Y)\right|}{\|Y\|}=0 .
$$

If $F$ is Fréchet differentiable at every $X_{0} \in \mathcal{E}, F$ is called Fréchet differentiable on $\mathcal{E}$ and the function $D F$ which assigns to every point $X_{0} \in \mathcal{E}$ the derivative $D F\left(X_{0}\right)$, is called the Fréchet derivative of the function $F$. If, in addition, the derivative $D F$ is continuous, $F$ is said to be a class $\mathcal{C}^{1}$-function, in symbols, $F \in \mathcal{C}^{1}(\mathcal{E}, \mathbb{R})$. 
Theorem 2.3. Let $G_{p}: S_{p} \rightarrow \mathbb{R}^{+}, 1<p<\infty, G_{p}(X)=\|X\|_{p}^{p}$, and let $X, Y \in S_{p}$. Then, for $1<p<\infty$, $G_{p}$ has a Fréchet derivative given by

$$
D G_{p}(X)(Y)=p \operatorname{Re}\left[\operatorname{tr}\left(|X|^{p-1} U^{*} Y\right)\right],
$$

where $\operatorname{Re}(z)$ is the real part of a complex number $z$ and $X=U|X|$ is the polar decomposition of the operator $X$, with $U$ the partial isometry such that $N(U)=N(X)$.

Proof. See [1, Theorem 2.1].

Observe that, if $p=2$. Then, $G_{2}(X)=\operatorname{tr}\left(X^{*} X\right)$ and

$$
D G_{2}(X)(Y)=2 R e \operatorname{tr}\left(X^{*} Y\right), X, Y \in S_{2} .
$$

\section{Global solutions of weighted least squares problems}

Given $A \in L(\mathcal{H}, \mathcal{F}), W \in L(\mathcal{F})^{+}$and $x \in \mathcal{F}$, a weighted least squares solution (or $W$-LSS) of the equation $A z=x$ is a vector $u \in \mathcal{H}$ such that

$$
\|A u-x\|_{W} \leq\|A z-x\|_{W}, \quad \text { for every } z \in \mathcal{H},
$$

where $\|x\|_{W}=\langle W x, x\rangle$ is the seminorm associated to $W$. The related problem is the classical weighted least squares problem.

If $W=I$, then problem (WLSP is the well-known least squares problem. Given $A \in L(\mathcal{H}, \mathcal{F})$ and $x \in \mathcal{F}$, it can be proved that $u$ is a least squares solution of $A z=x$ if and only if $A u-x \in R(A)^{\perp}$ (see [24, Theorem 3.1]). As a consequence, it is not difficult to prove the following result.

Proposition 3.1. Let $A \in L(\mathcal{H}, \mathcal{F}), W \in L(\mathcal{F})^{+}$and $x \in \mathcal{F}$. Then $u$ is $a W-L S S$ of $A z=x$ if and only if $A u-x \in W(R(A))^{\perp}$ or, equivalently, $A^{*} W(A u-x)=0$.

To study the existence of solutions of problem WLSP for every $x \in \mathcal{F}$ in the finite dimensional case, Rao and Mitra introduced the notion of $W$-inverse [26]. Later on, the $W$-inverse was studied for operators in [9] and [11].

Definition 1. Given $A \in L(\mathcal{H}, \mathcal{F})$ and $W \in L(\mathcal{F})^{+}$. An operator $G \in L(\mathcal{F}, \mathcal{H})$ is called a $W$-inverse of $A$ (or a bounded global solution of problem (WLSP) ) if for each $x \in \mathcal{F}, G x$ is a $W$-LSS of $A z=x$, i.e.

$$
\|A G x-x\|_{W} \leq\|A z-x\|_{W}, \quad \text { for every } z \in \mathcal{H} .
$$

Observe that $A$ has a $W$-inverse if the problem WLSP admits a solution for every $x \in \mathcal{F}$ and, moreover, it is possible to assign a $W$-LSS to each $x \in \mathcal{F}$ in a linear and continuous way.

The following result gives necessary and sufficient conditions for problem (WLSP) to admit a bounded global solution, when $A$ is not necessarily a closed range operator (cf. [1]]).

Theorem 3.2. Let $A \in L(\mathcal{H}, \mathcal{F})$ and $W \in L(\mathcal{F})^{+}$. Then the following statements are equivalent:

i) $A z=x$ admits a $W$-LSS for every $x \in \mathcal{F}$,

ii) $R(A)+W(R(A))^{\perp}=\mathcal{F}$,

iii) the normal equation

$$
A^{*} W(A X-I)=0
$$

admits a solution,

iv) the operator $A$ admits a $W$-inverse.

In this case, the set of $W$-inverses of $A$ is the set of solutions of (3.1). 
Proof. $\quad$ Along the proof we will use that $W(R(A))^{\perp}=N\left(A^{*} W\right)$.

i) $\Rightarrow$ ii) : By Proposition 3.1. Problem (WLSP admits a solution for every $x \in \mathcal{F}$ if and only if there exists $u$ such that $A^{*} W A u=A^{*} W x$ for every $x \in \mathcal{F}$ or, equivalently $R\left(A^{*} W\right)=R\left(A^{*} W A\right)$. Then

$$
\mathcal{F}=\left(A^{*} W\right)^{-1}\left(R\left(A^{*} W\right)\right)=\left(A^{*} W\right)^{-1}\left(A^{*} W(R(A))\right)=R(A)+W(R(A))^{\perp} .
$$

ii $) \Rightarrow$ iii $)$ : Suppose that $R(A)+W(R(A))^{\perp}=\mathcal{F}$. Then $R\left(A^{*} W\right)=R\left(A^{*} W A\right)$ and the assertion follows by applying Douglas' Lemma [15].

iii) $\Rightarrow$ iv) : There exists $X_{0} \in L(\mathcal{F}, \mathcal{H})$ such that $A^{*} W A X_{0}=A^{*} W$ if and only if $A^{*} W\left(A X_{0} x-x\right)=0$ for every $x \in \mathcal{F}$ or $A X_{0} x-x \in W(R(A))^{\perp}$ for every $x \in \mathcal{F}$. Therefore, by Proposition 3.1 $X_{0}$ is a $W$ inverse of $A$.

$i v) \Rightarrow i):$ It is straightforward.

In this case, we have also proved that the set of $W$-inverses of $A$ is the set of solutions of (3.1).

Corollary 3.3. Let $A \in L(\mathcal{H}, \mathcal{F})$ and $W \in L(\mathcal{F})^{+}$. If $A$ admits a $W$-inverse then $(W, \overline{R(A)})$ is compatible.

Proof. If $A$ admits a $W$-inverse then, by Theorem 3.2 and the identity $W(R(A))^{\perp}=W(\overline{R(A)})^{\perp}$, we get that

$$
\mathcal{F}=R(A)+W(R(A))^{\perp} \subseteq \overline{R(A)}+W(\overline{R(A)})^{\perp} .
$$

Therefore, by Proposition 2.1, the pair $(W, \overline{R(A)})$ is compatible.

A non closed range operator can admit a $W$-inverse, as the following example shows.

Example 1. Consider $W \in L(\mathcal{F})^{+}$with infinite dimensional nullspace and $A=P_{N(W)^{\perp}}+A_{2} P_{N(W)}$, where $A_{2} \in L(N(W))$ and $R\left(A_{2}\right)$ is not closed. Then $R(A)$ is not closed and

$$
R(A)+W(R(A))^{\perp}=R(A)+W\left(N(W)^{\perp}\right)^{\perp}=R(A)+R(W)^{\perp} \supseteq N(W)^{\perp}+R(W)^{\perp}=\mathcal{F},
$$

so that, by Theorem 3.2, it holds that $A$ admits a $W$-inverse.

If $W=I$, it is well known that the least squares problem $A z=x$ admits a solution for every $x \in \mathcal{F}$ if and only if $R(A)$ is closed. More generally:

Proposition 3.4. Let $A \in L(\mathcal{H}, \mathcal{F})$ and $W \in L(\mathcal{F})^{+}$. Then $A$ admits a $W$-inverse if and only if $R(A)+$ $N(W)$ is closed and the pair $(W, R(A)+N(W))$ is compatible.

Proof. $\quad$ Suppose that $A$ admits a $W$-inverse. Then, by Theorem 3.2, it holds that $\mathcal{F}=R(A)+$ $W(R(A))^{\perp}=R(A)+W^{-1}\left((R(A))^{\perp}\right)$. Applying $W^{1 / 2}$, it follows that $R\left(W^{1 / 2}\right)=W^{1 / 2}(R(A))+$ $W^{1 / 2}\left(W^{-1}\left(R(A)^{\perp}\right)\right)=W^{1 / 2}(R(A))+W^{1 / 2}(R(A))^{\perp} \cap R\left(W^{1 / 2}\right)$. Therefore, $W^{1 / 2}(R(A))$ is closed in $R\left(W^{1 / 2}\right)$. Hence, $R(A)+N(W)=R(A)+N\left(W^{1 / 2}\right)=W^{-1 / 2}\left(W^{1 / 2}(R(A))\right.$ is closed. Since $\mathcal{F}=$ $R(A)+W(R(A))^{\perp}=R(A)+N(W)+W(R(A)+N(W))^{\perp}$, by Proposition 2.1, the pair $(W, R(A)+N(W))$ is compatible. Conversely, suppose that $R(A)+N(W)$ is closed and the pair $(W, R(A)+N(W))$ is compatible. Then $\mathcal{F}=R(A)+N(W)+W(R(A)+N(W))^{\perp}=R(A)+W(R(A))^{\perp}$, because $N(W) \subseteq W(R(A))^{\perp}$. Therefore, by Theorem 3.2 , $A$ admits a $W$-inverse.

Proposition 3.5. Let $A \in L(\mathcal{H}, \mathcal{F})$ and $W \in L(\mathcal{F})^{+}$. If $A$ admits a $W$-inverse then $R(A)$ is closed if and only if $R(A) \cap N(W)$ is closed.

Proof. If $A$ admits a $W$-inverse then, by Theorem 3.2 it holds that $\mathcal{F}=R(A)+W(R(A))^{\perp}$. Since $W \in L(\mathcal{F})^{+}$, it is not difficult to see that $R(A) \cap W(R(A))^{\perp}=R(A) \cap N(W)$. Suppose that $R(A) \cap N(W)$ is closed, then $\mathcal{F}=R(A)+W(R(A))^{\perp}=R(A) \dot{+}\left[W(R(A))^{\perp} \ominus(R(A) \cap N(W))\right]$. Hence, by [18, Theorem 2.3 , it follows that $R(A)$ is closed. The converse is straightforward. 


\section{Operator weighted least squares problems}

In this subsection we are interested in studying weighted least squares problems for operators considering Schatten $p$-norms.

Given $A \in L(\mathcal{H}, \mathcal{F})$ and $W \in L(\mathcal{F})^{+}$such that $W^{1 / 2} \in S_{p}$ for some $1 \leq p<\infty$, the problem is to determine if there exists

$$
\min _{X \in L(\mathcal{F}, \mathcal{H})}\|A X-I\|_{p, W}
$$

where $\|Y\|_{p, W}=\left\|W^{1 / 2} Y\right\|_{p}$, for $Y \in L(\mathcal{F})$.

We will refer to problem (OWLSP as the operator weighted least squares problem.

In order to study problem (OWLSP), we introduce the following associated problem: given $A \in$ $L(\mathcal{H}, \mathcal{F})$ and $W \in L(\mathcal{F})^{+}$, analyze the existence of

$$
\min _{X \in L(\mathcal{F}, \mathcal{H})}(A X-I)^{*} W(A X-I),
$$

in the order induced in $L(\mathcal{F})$ by the cone of positive operators. By studying problems (OWLSP and (3.2) we will relate the existence of solutions of (OWLSP to the existence of bounded global solutions of (WLSP).

In [1], problems (OWLSP) and (3.2) were studied for $A \in L(\mathcal{H})$ such that $R(A)$ is closed. The results obtained in [11] are also valid in the general case.

Proposition 3.6. Let $A \in L(\mathcal{H}, \mathcal{F})$ and $W \in L(\mathcal{F})^{+}$such that $W^{1 / 2} \in S_{p}$ for some $1 \leq p<\infty$. Then the following statements are equivalent:

i) there exists $\min _{X \in L(\mathcal{F}, \mathcal{H})}\|A X-I\|_{p, W}$,

ii) $R(A)+W(R(A))^{\perp}=\mathcal{F}$,

iii) there exists $\min _{X \in L(\mathcal{F}, \mathcal{H})}(A X-I)^{*} W(A X-I)$.

In this case,

$$
\min _{X \in L(\mathcal{F}, \mathcal{H})}\|A X-I\|_{p, W}=\left\|W_{/ \overline{R(A)}}^{1 / 2}\right\|_{p} \text { and } \min _{X \in L(\mathcal{F}, \mathcal{H})}(A X-I)^{*} W(A X-I)=W_{/ \overline{R(A)}} .
$$

Proof. The equivalence between $i$ ), ii) and iii) follows by similar arguments as those in the proofs of 11, Theorem 4.3] and [11, Theorem 4.5], using Proposition 3.1.

In this case. Let $X_{0}$ be a solution of problem (3.2), i.e., $\left(A X_{0}-I\right)^{*} W\left(A X_{0}-I\right)=\min _{X \in L(\mathcal{F}, \mathcal{H})}(A X-$ $I)^{*} W(A X-I)$. Then, in particular, $0 \leq\left(A X_{0}-I\right)^{*} W\left(A X_{0}-I\right) \leq W$ and, by similar arguments as those found in [11, Proposition 4.4], $A^{*} W\left(A X_{0}-I\right)=0$. Therefore, since $A^{*}\left[\left(A X_{0}-I\right)^{*} W\left(A X_{0}-I\right)\right]=$ $A^{*} X_{0}^{*} A^{*} W\left(A X_{0}-I\right)-A^{*} W\left(A X_{0}-I\right)=0$, we have that $R\left(\left(A X_{0}-I\right)^{*} W\left(A X_{0}-I\right)\right) \subseteq R(A)^{\perp}$. Let $Z \in L(\mathcal{F})^{+}$such that $Z \leq W$ and $R(Z) \subseteq R(A)^{\perp}$. Then,

$$
Z=\left(A X_{0}-I\right)^{*} Z\left(A X_{0}-I\right) \leq\left(A X_{0}-I\right)^{*} W\left(A X_{0}-I\right) .
$$

Therefore $\min _{X \in L(\mathcal{F}, \mathcal{H})}(A X-I)^{*} W(A X-I)=\left(A X_{0}-I\right)^{*} W\left(A X_{0}-I\right)=\max \{Z \in L(\mathcal{F}): 0 \leq Z \leq$ $W$ and $\left.R(Z) \subseteq R(A)^{\perp}\right\}=W_{/ \overline{R(A)}}$.

Finally, by Proposition 2.2. $\left\|A X_{0}-I\right\|_{p, W}=\left\|W_{/ / R(A)}^{1 / 2}\right\|_{p}$ and

$$
\min _{X \in L(\mathcal{F}, \mathcal{H})}\|A X-I\|_{p, W}=\left\|A X_{0}-I\right\|_{p, W}=\left\|W_{/ \overline{R(A)}}^{1 / 2}\right\|_{p}
$$

The next corollary summarizes the results of the section. 
Corollary 3.7. Let $A \in L(\mathcal{H}, \mathcal{F})$ and $W \in L(\mathcal{F})^{+}$such that $W^{1 / 2} \in S_{p}$ for some $1 \leq p<\infty$. Then the following statements are equivalent:

i) $A z=x$ admits a $W$-LSS for every $x \in \mathcal{F}$,

ii) A admits a $W$-inverse, i.e., for every $x \in \mathcal{F}, A z=x$ admits a $W$-LSS $G x$ with $G \in L(\mathcal{F}, \mathcal{H})$,

iii) there exists $\min _{X \in L(\mathcal{F}, \mathcal{H})}\|A X-I\|_{p, W}$.

\section{Global solutions of spline and smoothing problems}

Following similar ideas as those presented in Section 3, we will study under which conditions the classical spline and smoothing problems admit global solutions. Moreover, we will relate bounded global solutions to the solutions of the associated operator minimization problems.

Splines problems

Let $T \in L(\mathcal{H}, \mathcal{E}), V \in L(\mathcal{H}, \mathcal{F})$ and $f_{0} \in R(V)$, we study the existence of

$$
\min \|T h\| \text {, subject to } V h=f_{0} .
$$

Suppose that $V h_{0}=f_{0}$, problem (4.1) is equivalent to study when the set

$$
\operatorname{sp}\left(T, N(V), h_{0}\right)=\left\{h \in h_{0}+N(V):\|T h\|=\min _{z \in N(V)}\left\|T\left(h_{0}+z\right)\right\|\right\}
$$

is not empty. We will refer to problem (SP) as the classical spline problem and any element of the set $s p\left(T, N(V), h_{0}\right)$ is an abstract spline or a $(T, N(V))$-spline interpolant to $h_{0}$.

In order to obtain solutions of $(\mathrm{SP})$ that depend continuously on $h$ we give the following definition.

Definition 2. Let $T \in L(\mathcal{H}, \mathcal{E})$ and $V \in L(\mathcal{H}, \mathcal{F})$. An operator $G \in L(\mathcal{H})$ is a bounded global solution of (SP) if

$$
G h \in \operatorname{sp}(T, N(V), h) \text { for every } h \in \mathcal{H} .
$$

We are also interested in comparing the bounded global solution of (SP) to the operator spline problem: given $T \in S_{p}(\mathcal{H}, \mathcal{E})$ for some $1 \leq p<\infty, V \in L(\mathcal{H}, \mathcal{F})$ and $B_{0} \in L(\mathcal{H}, \mathcal{F})$ such that $R(V) \subseteq R\left(B_{0}\right)$, analyze the existence of

$$
\min _{V X=B_{0}}\|T X\|_{p}
$$

where $X \in L(\mathcal{H})$.

We begin by studying problem (OSP). The next result characterizes the existence of solutions of (OSP) and describes the operators where the minimum is attained.

Proposition 4.1. Let $T \in S_{p}(\mathcal{H}, \mathcal{E})$ for some $1 \leq p<\infty, V \in L(\mathcal{H}, \mathcal{F})$ and $B_{0} \in L(\mathcal{H}, \mathcal{F})$ such that $R\left(B_{0}\right) \subseteq R(V)$. Then the following statements are equivalent:

i) there exists $\min _{V X=B_{0}}\|T X\|_{p}$,

ii) $R\left(V^{\dagger} B_{0}\right) \subseteq N(V)+\left[T^{*} T(N(V))\right]^{\perp}$,

iii) the normal equation

$$
P_{N(V)} T^{*} T\left(P_{N(V)} X+V^{\dagger} B_{0}\right)=0
$$

admits a solution. 
In this case,

$$
\min _{V X=B_{0}}\|T X\|_{p}=\left\|\left[\left(T^{*} T\right)_{/ N(V)}\right]^{1 / 2} V^{\dagger} B_{0}\right\|_{p}=\left\|T X_{0}\right\|_{p}
$$

where $X_{0}$ is any solution of equation (4.3).

Proof. $\quad i) \Leftrightarrow i i$ ) : Note that if $V X=B_{0}$ then $V^{\dagger} V X=P_{N(V)^{\perp}} X=V^{\dagger} B_{0} \in L(\mathcal{H})$ (see Douglas' Lemma [15]). Then, $X=P_{N(V)} X+V^{\dagger} B_{0}$ and, since $\|T X\|_{p}=\|X\|_{p, T * T}$, we get that

$$
\min _{V X=B_{0}}\|T X\|_{p}=\min _{X \in L(\mathcal{H})}\left\|P_{N(V)} X+V^{\dagger} B_{0}\right\|_{p, T^{*} T} .
$$

Then, by [11, Theorem 4.5], problem (OSP) admits a solution if only if

$$
\mathrm{R}\left(V^{\dagger} B_{0}\right) \subseteq N(V)+\left[T^{*} T(N(V))\right]^{\perp} .
$$

ii) $\Leftrightarrow$ iii) : It follows from [11, Theorem 2.4].

In this case, by [11, Theorem 4.5] and [11, Theorem 2.4],

$$
\min _{V X=B_{0}}\|T X\|_{p}=\left\|\left[\left(T^{*} T\right) / N(V)\right]^{1 / 2} V^{\dagger} B_{0}\right\|_{p}=\left\|T X_{0}\right\|_{p}
$$

where $X_{0}$ is any solution of equation (4.3).

Proposition 4.2. Let $T \in S_{p}(\mathcal{H}, \mathcal{E})$ for some $1 \leq p<\infty, V \in L(\mathcal{H}, \mathcal{F})$ and $B_{0} \in L(\mathcal{H}, \mathcal{F})$ such that $R\left(B_{0}\right) \subseteq R(V)$. Then $X_{0} \in L(\mathcal{H})$ is a solution of (OSP) if and only if

$$
X_{0} x \in \operatorname{sp}\left(T, N(V), V^{\dagger} B_{0} x\right) \text { for every } x \in \mathcal{H} .
$$

Proof. Suppose $X_{0} \in L(\mathcal{H})$ is a solution of $\mathrm{OSP}$. Then there exists $Y_{0} \in L(\mathcal{H})$ such that $X_{0}=$ $P_{N(V)} Y_{0}+V^{\dagger} B_{0}$ and $X_{0}$ is a solution of (4.3). Then, $Y_{0}$ is a solution of (4.3) too. So, by 11, Proposition 4.4],

$$
\left(P_{N(V)} Y_{0}+V^{\dagger} B_{0}\right)^{*} T^{*} T\left(P_{N(V)} Y_{0}+V^{\dagger} B_{0}\right) \leq\left(P_{N(V)} Y+V^{\dagger} B_{0}\right)^{*} T^{*} T\left(P_{N(V)} Y+V^{\dagger} B_{0}\right),
$$

for every $Y \in L(\mathcal{H})$. Or, equivalently,

$$
\left\|T\left(P_{N(V)} Y_{0}+V^{\dagger} B_{0}\right) x\right\| \leq\left\|T\left(P_{N(V)} Y+V^{\dagger} B_{0}\right) x\right\| \text {, for every } x \in \mathcal{H} \text { and } Y \in L(\mathcal{H}) .
$$

Let $z \in \mathcal{H}$ be arbitrary. For every $x \in \mathcal{H} \backslash\{0\}$, there exists $Y \in L(\mathcal{H})$ such that $z=Y x$. Therefore

$$
\left\|T\left(P_{N(V)} Y_{0}+V^{\dagger} B_{0}\right) x\right\| \leq\left\|T\left(P_{N(V)} z+V^{\dagger} B_{0} x\right)\right\|, \text { for every } x, z \in \mathcal{H} .
$$

Then $X_{0} x \in V^{\dagger} B_{0} x+N(V)$,

$$
\left\|T X_{0} x\right\| \leq\|T h\|, \text { for every } h \in V^{\dagger} B_{0} x+N(V)
$$

and $X_{0} x \in \operatorname{sp}\left(T, N(V), V^{\dagger} B_{0} x\right)$.

Conversely, suppose that $X_{0} x \in \operatorname{sp}\left(T, N(V), V^{\dagger} B_{0} x\right)$ for every $x \in \mathcal{H}$. Then, for every $x \in \mathcal{H}, X_{0} x \in$ $V^{\dagger} B_{0} x+N(V)$ and

$$
\left\|T X_{0} x\right\| \leq\|T h\|, \text { for every } h \in V^{\dagger} B_{0} x+N(V) .
$$

It follows that $V X_{0}=B_{0}$ and

$$
\left\|T X_{0} x\right\| \leq\left\|T\left(P_{N(V)} z+V^{\dagger} B_{0} x\right)\right\|, \text { for every } x, z \in \mathcal{H} .
$$

In particular, given $Y \in L(\mathcal{H})$, consider $z=Y x$. Then

$$
\left\|T X_{0} x\right\| \leq\left\|T\left(P_{N(V)} Y+V^{\dagger} B_{0}\right) x\right\| \text { for every } x \in \mathcal{H} \text { and } Y \in L(\mathcal{H}),
$$


or, equivalently,

$$
X_{0}^{*} T^{*} T X_{0} \leq\left(P_{N(V)} Y+V^{\dagger} B_{0}\right)^{*} T^{*} T\left(P_{N(V)} Y+V^{\dagger} B_{0}\right) \text {, for every } Y \in L(\mathcal{H}) .
$$

Then, by Proposition 2.2

$$
\left\|T X_{0}\right\|_{p}=\left\||T| X_{0}\right\|_{p} \leq\left\||T|\left(P_{N(V)} Y+V^{\dagger} B_{0}\right)\right\|_{p}=\left\|P_{N(V)} X+V^{\dagger} B_{0}\right\|_{p, T^{*} T}
$$

for every $Y \in L(\mathcal{H})$. Therefore, $X_{0}$ is a solution of (OSP).

The following result gives necessary and sufficient conditions for the operator spline problem (OSP) to have a solution for every $B_{0} \in L(\mathcal{H}, \mathcal{F})$. Moreover, it shows that this is equivalent to the condition that guarantees the existence of a bounded global solution of the classical spline problem (SP).

Theorem 4.3. Let $T \in S_{p}(\mathcal{H}, \mathcal{E})$ for some $1 \leq p<\infty$ and $V \in L(\mathcal{H}, \mathcal{F})$. Then the following statements are equivalent:

i) there exists $\min _{V X=B_{0}}\|T X\|_{p}$ for every $B_{0} \in L(\mathcal{H}, \mathcal{F})$ such that $R\left(B_{0}\right) \subseteq R(V)$,

ii) there exists a bounded global solution of (SP),

iii) the pair $\left(T^{*} T, N(V)\right)$ is compatible,

iv) $\operatorname{sp}\left(T, N(V), h_{0}\right)$ is nonempty for every $h_{0} \in \mathcal{H}$.

Proof. $\quad i) \Leftrightarrow i i)$ : Suppose that $X_{0} \in L(\mathcal{H})$ is a solution of $\mathrm{OSP}$ for $B_{0}=V$. Consider $G:=$ $X_{0} P_{N(V)^{\perp}} \in L(\mathcal{H})$. Then, by Proposition 4.2.

$$
G h=X_{0}\left(P_{N(V)^{\perp}} h\right) \in \operatorname{sp}\left(T, N(V), P_{N(V)^{\perp}} h\right) \text { for every } h \in \mathcal{H} .
$$

Note that $s p\left(T, N(V), P_{N(V)^{\perp}} h\right)=s p(T, N(V), h)$, because $P_{N(V)^{\perp}} h+N(V)=h+N(V)$. Hence,

$$
G h \in \operatorname{sp}(T, N(V), h) \text { for every } h \in \mathcal{H},
$$

so that $G$ is a bounded global solution of (SP).

Conversely, suppose that $G \in L(\mathcal{H})$ is a bounded global solution of $(\mathrm{SP})$ and $B_{0} \in L(\mathcal{H}, \mathcal{F})$. Set $X_{0}:=G V^{\dagger} B_{0} \in L(\mathcal{H})$, then

$$
X_{0} x=G\left(V^{\dagger} B_{0} x\right) \in \operatorname{sp}\left(T, N(V), V^{\dagger} B_{0} x\right) \text { for every } x \in \mathcal{H} .
$$

Therefore, by Proposition 4.2, $X_{0}$ is a solution of (OSP).

$i) \Leftrightarrow i$ ii $)$ : Suppose that (OSP has a solution for every $B_{0} \in L(\mathcal{H}, \mathcal{F})$. Then, by Proposition 4.1.

$$
\mathrm{R}\left(V^{\dagger} B_{0}\right) \subseteq N(V)+\left[T^{*} T(N(V)]^{\perp}\right.
$$

for every $B_{0}$ such that $R\left(B_{0}\right) \subseteq R(V)$. Consider $B_{0}$ such that $R\left(B_{0}\right)=R(V)$, then $N(V)^{\perp}=\mathrm{R}\left(V^{\dagger} B_{0}\right) \subseteq$ $N(V)+\left[T^{*} T(N(V)]^{\perp}\right.$, so that $\mathcal{H}=N(V)+\left[T^{*} T(N(V)]^{\perp}\right.$ and, by Proposition 2.1, the pair $\left(T^{*} T, N(V)\right)$ is compatible.

Conversely, let the pair $\left(T^{*} T, N(V)\right)$ be compatible. By Proposition 2.1. $\mathcal{H}=N(V)+\left[T^{*} T(N(V)]^{\perp}\right.$. Then, for every $B_{0}, \mathrm{R}\left(V^{\dagger} B_{0}\right) \subseteq N(V)+\left[T^{*} T(N(V)]^{\perp}\right.$ and, by Proposition 4.1, (OSP has a solution for every $B_{0} \in L(\mathcal{H}, \mathcal{F})$ such that $R\left(B_{0}\right) \subseteq R(V)$.

iii) $\Leftrightarrow i v)$ : See [14, Theorem 3.2]. 


\section{Smoothing problems}

Let $T \in L(\mathcal{H}, \mathcal{E}), V \in L(\mathcal{H}, \mathcal{F})$ and $f_{0} \in \mathcal{F}$. A problem that is naturally associated with $(\mathrm{SP})$ is to find

$$
\min _{h \in \mathcal{H}}\left(\|T h\|^{2}+\left\|V h-f_{0}\right\|^{2}\right) .
$$

We will refer to (SMP) as the classical smoothing problem and its solutions are called smoothing splines.

As before, we also study the problem of finding a bounded global solution of problem (SMP); i.e., we analyze if there exists an operator $G \in L(\mathcal{F}, \mathcal{H})$ such that

$$
\|T G f\|^{2}+\|V G f-f\|^{2}=\min _{h \in \mathcal{H}}\left(\|T h\|^{2}+\|V h-f\|^{2}\right) \text {, for every } f \in \mathcal{F} .
$$

Several properties of bounded global solutions of problem (SMP) were given in [10, Section 4] for $V \in L(\mathcal{H}, \mathcal{F})$ with closed range.

We are interested in characterizing bounded global solutions of (SMP) in the general case and comparing them with the solutions of the following operator smoothing problem: given $T \in S_{2}(\mathcal{H}, \mathcal{E})$, $V \in S_{2}(\mathcal{H}, \mathcal{F})$ and $B_{0} \in S_{2}(\mathcal{H}, \mathcal{F})$, analyze the existence of

$$
\min _{X \in L(\mathcal{H})}\left(\|T X\|_{2}^{2}+\left\|V X-B_{0}\right\|_{2}^{2}\right) .
$$

Define $K, B_{0}^{\prime}: \mathcal{H} \rightarrow \mathcal{E} \oplus \mathcal{F}$

$$
\begin{array}{r}
K h=(T h, V h) \text { for } h \in \mathcal{H}, \\
B_{0}^{\prime} h=\left(0, B_{0} h\right) \text { for } h \in \mathcal{H} .
\end{array}
$$

We will consider the inner product and the associated norm on $\mathcal{E} \oplus \mathcal{F}$ as in 2.1). It is straightforward to check that the adjoint of $K, K^{*}: \mathcal{E} \oplus \mathcal{F} \rightarrow \mathcal{H}$, is $K^{*}(e, f)=T^{*} h+V^{*} f$, for $e \in \mathcal{E}$ and $f \in \mathcal{F}$ and the adjoint of $B_{0}^{\prime}, B_{0}^{\prime *}: \mathcal{E} \oplus \mathcal{F} \rightarrow \mathcal{H}$, is $B_{0}^{\prime *}(e, f)=B_{0}^{*} f$, for $e \in \mathcal{E}$ and $f \in \mathcal{F}$.

Lemma 4.4. Let $T \in L(\mathcal{H}, \mathcal{E}), V \in L(\mathcal{H}, \mathcal{F})$ and $B_{0} \in L(\mathcal{H}, \mathcal{F})$. Set $K$ and $B_{0}^{\prime}$ as in (4.6) and (4.7). Then there exists $X_{0} \in L(\mathcal{H})$ such that

$$
\left(K X_{0}-B_{0}^{\prime}\right)^{*}\left(K X_{0}-B_{0}^{\prime}\right)=\min _{X \in L(\mathcal{H})}\left(K X-B_{0}^{\prime}\right)^{*}\left(K X-B_{0}^{\prime}\right),
$$

where the order is the one induced in $L(\mathcal{H})$ by the cone of positive operators, if and only if $X_{0}$ is a solution of the normal equation

$$
\left(T^{*} T+V^{*} V\right) X=V^{*} B_{0} .
$$

Proof. This follows in a similar way as in the proof of Proposition 3.6 and Theorem 3.2 using the fact that $u$ is a least squares solution of the equation $K z=B_{0}^{\prime} x$ for every $x \in \mathcal{H}$ if and only if $u$ is a solution of $K^{*}\left(K y-B_{0}^{\prime} x\right)=0$, see Proposition 3.1

Proposition 4.5. Let $T \in S_{2}(\mathcal{H}, \mathcal{E}), V \in S_{2}(\mathcal{H}, \mathcal{F})$ and $B_{0} \in S_{2}(\mathcal{H}, \mathcal{F})$. Then the following statements are equivalent:

i) there exists $\min _{X \in L(\mathcal{H})}\left(\|T X\|_{2}^{2}+\left\|V X-B_{0}\right\|_{2}^{2}\right)$,

ii) the normal equation $\left(T^{*} T+V^{*} V\right) X=V^{*} B_{0}$ admits a solution.

Proof. Let $K$ and $B_{0}^{\prime}$ be as in (4.6) and (4.7). If $X_{0}$ is a solution of the normal equation (4.8), then by Lemma 4.4. $\left(K X_{0}-B_{0}^{\prime}\right)^{*}\left(K X_{0}-B_{0}^{\prime}\right) \leq\left(K X-B_{0}^{\prime}\right)^{*}\left(K X-B_{0}^{\prime}\right)$, for every $X \in L(\mathcal{H})$. Observe that, for every $X \in L(\mathcal{H}),\left|\left(K X-B_{0}^{\prime}\right)\right|^{2}=\left(K X-B_{0}^{\prime}\right)^{*}\left(K X-B_{0}^{\prime}\right)=|T X|^{2}+\left|V X-B_{0}\right|^{2}$. Then, for every $X \in L(\mathcal{H})$

$$
\left\|T X_{0}\right\|_{2}^{2}+\left\|V X_{0}-B_{0}\right\|_{2}^{2}=\operatorname{tr}\left(\left|\left(K X_{0}-B_{0}^{\prime}\right)\right|^{2}\right) \leq \operatorname{tr}\left(\left|\left(K X-B_{0}^{\prime}\right)\right|^{2}\right)=\|T X\|_{2}^{2}+\left\|V X-B_{0}\right\|_{2}^{2},
$$


see Proposition 2.2. Thus, OSMP admits a solution.

To prove the converse, consider $F_{2}: L(\mathcal{H}) \rightarrow \mathbb{R}^{+}$,

$$
\begin{aligned}
F_{2}(X) & =\|T X\|_{2}^{2}+\left\|V X-B_{0}\right\|_{2}^{2}=\|T X\|_{2}^{2}+\operatorname{tr}\left(\left(V X-B_{0}\right)^{*}\left(V X-B_{0}\right)\right) \\
& =\|T X\|_{2}^{2}+\|V X\|_{2}^{2}-2 \operatorname{Re}\left[\operatorname{tr}\left(B_{0}^{*} V X\right)\right]+\left\|B_{0}\right\|_{2}^{2} \\
& =\left\|T\left|X\left\|_{2}^{2}+\right\|\right| V \mid X\right\|_{2}^{2}-2 \operatorname{Re}\left[\operatorname{tr}\left(B_{0}^{*} V X\right)\right]+\left\|B_{0}\right\|_{2}^{2}
\end{aligned}
$$

By Theorem 2.3. $F_{2}$ has a Fréchet derivative and, furthermore, for every $X, Y \in L(\mathcal{H})$

$$
\begin{aligned}
D F_{2}(X)(Y) & =D G_{2}(|T| X)(|T| Y)+D G_{2}(|V| X)(|V| Y)-2 R e\left[\operatorname{tr}\left(B_{0}^{*} V Y\right)\right] \\
& =2 R e\left[\operatorname{tr}\left(\left(|T| X_{0}\right)^{*}|T| Y\right)\right]+2 R e\left[\operatorname{tr}\left(\left(|V| X_{0}\right)^{*}|V| Y\right)\right]-2 R e\left[\operatorname{tr}\left(B_{0}^{*} V Y\right)\right],
\end{aligned}
$$

where $G_{2}(X)=\|X\|_{2}^{2}=\operatorname{tr}\left(X^{*} X\right)$.

Suppose that $X_{0} \in L(\mathcal{H})$ is a global minimum of $\|T X\|_{2}^{2}+\left\|V X-B_{0}\right\|_{2}^{2}$. Then $X_{0}$ is a global minimum of $F_{2}$ and, since $F_{2}$ is a $\mathcal{C}^{1}$-function

$$
D F_{2}\left(X_{0}\right)(Y)=0 \text {, for every } Y \in L(\mathcal{H}) .
$$

Then, for every $Y \in L(\mathcal{H})$,

$$
\operatorname{Re}\left[\operatorname{tr}\left(\left(\left(|T| X_{0}\right)^{*}|T|+\left(|V| X_{0}\right)^{*}|V|-B_{0}^{*} V\right) Y\right)\right]=0 .
$$

Then, it follows that

$$
X_{0}^{*}|T|^{2}+X_{0}^{*}|V|^{2}-B_{0}^{*} V=0
$$

or, equivalently

$$
\left(T^{*} T+V^{*} V\right) X_{0}=V^{*} B_{0} .
$$

To study the existence of solutions of inconsistent linear systems under seminorms defined by positive semidefinite matrices, Mitra defined the optimal inverses for matrices [23]. In [10], Mitra's concept was extended to Hilbert spaces:

Definition 3. Given operators $A \in L(\mathcal{H}, \mathcal{F})$ and $W \in L(\mathcal{F} \oplus \mathcal{H})^{+}$, a $W$-optimal inverse of $A$ is an operator $G \in L(\mathcal{F}, \mathcal{H})$ such that

$$
\left\|\left(\begin{array}{c}
A G f-f \\
G f
\end{array}\right)\right\|_{W}=\min _{h \in \mathcal{H}}\left\|\left(\begin{array}{c}
A h-f \\
h
\end{array}\right)\right\|_{W},
$$

for every $f \in \mathcal{F}$. Here $\|\cdot\|_{W}$ denotes the seminorm defined by $W:\left\|\left(\begin{array}{c}f \\ h\end{array}\right)\right\|_{W}=\left\|W^{1 / 2}\left(\begin{array}{c}f \\ h\end{array}\right)\right\|$.

Consider $W$ with the following block form

$$
\left(\begin{array}{ll}
W_{11} & W_{12} \\
W_{12}^{*} & W_{22}
\end{array}\right)
$$

where $W_{11} \in L(\mathcal{F})^{+}, W_{22} \in L(\mathcal{H})^{+}$and $W_{12} \in L(\mathcal{H}, \mathcal{F})$. By [10, Theorem 2.1] and 23, Theorem 4.2], $A \in L(\mathcal{H}, \mathcal{F})$ admits a $W$-optimal inverse if and only if the equation

$$
\left(A^{*} W_{11} A+A^{*} W_{12}+W_{12}^{*} A+W_{22}\right) X=A^{*} W_{11}+W_{12}^{*}
$$

admits a solution. In this case, the set of $W$-optimal inverses of $A$ is the set of solutions of (4.10).

The following result relates the existence of a $W$-optimal inverse to the existence of a solution of OSMP). Some equivalences of the next proposition were proven in [10, Theorem 4.2] for $V \in L(\mathcal{H}, \mathcal{F})$ with closed range. The proofs of such equivalences are included in order to remark that the range of $V$ need not be closed. 
Theorem 4.6. Let $T \in S_{2}(\mathcal{H}, \mathcal{E})$ and $V \in S_{2}(\mathcal{H}, \mathcal{F})$. Then the following are equivalent:

i) there exists $\min _{X \in L(\mathcal{H})}\left(\|T X\|_{2}^{2}+\left\|V X-B_{0}\right\|_{2}^{2}\right)$ for every $B_{0} \in S_{2}(\mathcal{H}, \mathcal{F})$,

ii) $R\left(V^{*}\right) \subseteq R\left(T^{*} T+V^{*} V\right)$,

iii) there exists $\min _{h \in \mathcal{H}}\left(\|T h\|^{2}+\left\|V h-f_{0}\right\|^{2}\right)$ for every $f_{0} \in \mathcal{F}$,

iv) $V$ admits a $\left(\begin{array}{cc}I & 0 \\ 0 & T^{*} T\end{array}\right)$-optimal inverse,

v) there exists a bounded global solution of the classical smoothing problem (SMP).

If $R(V)$ is closed, conditions i) to $v$ ) are also equivalent to

vi) the pair $\left(T^{*} T, N(V)\right)$ is compatible.

Proof. $i) \Leftrightarrow i i)$ : Suppose that (OSMP has a minimum for every $B_{0} \in S_{2}(\mathcal{H}, \mathcal{F})$. Then, by Proposition 4.5 and Douglas' Lemma, $R\left(V^{*} B_{0}\right) \subseteq R\left(T^{*} T+V^{*} V\right)$. Consider $f_{0} \in \mathcal{F}$, then there exists $B_{0} \in S_{2}(\mathcal{H}, \mathcal{F})$ such that $f_{0}=B_{0} x$, for some $x \in \mathcal{H}$. Therefore

$$
V^{*} f_{0}=V^{*} B_{0} x \in R\left(V^{*} B_{0}\right) \subseteq R\left(T^{*} T+V^{*} V\right) .
$$

Hence $R\left(V^{*}\right) \subseteq R\left(T^{*} T+V^{*} V\right)$. Conversely, suppose that $R\left(V^{*}\right) \subseteq R\left(T^{*} T+V^{*} V\right)$ and consider $B_{0} \in$ $S_{2}(\mathcal{H}, \mathcal{F})$. Then $R\left(V^{*} B_{0}\right) \subseteq R\left(V^{*}\right) \subseteq R\left(T^{*} T+V^{*} V\right)$. Hence, by Douglas' Lemma and Proposition 4.5 . OSMP has a solution for every $B_{0} \in S_{2}(\mathcal{H}, \mathcal{F})$.

$i i) \Leftrightarrow i i i):$ Let $K$ be as in (4.6). Given $f_{0} \in \mathcal{F}$, it holds that $\min _{h \in \mathcal{H}}\left(\|T h\|^{2}+\left\|V h-f_{0}\right\|^{2}\right)=\min _{h \in \mathcal{H}} \| K h-$ $\left(0, f_{0}\right) \|^{2}$ exists if and only if the normal equation $K^{*} K h=K^{*}\left(0, f_{0}\right)$ has a solution; equivalently $V^{*} f_{0}=$ $\left(T^{*} T+V^{*} V\right) h$ has a solution. Therefore, $\min _{h \in \mathcal{H}}\left(\|T h\|^{2}+\left\|V h-f_{0}\right\|^{2}\right)$ exists for every $f_{0} \in \mathcal{H}$ if and only if $R\left(V^{*}\right) \subseteq R\left(T^{*} T+V^{*} V\right)$.

ii) $\Leftrightarrow i v)$ : It follows by (4.10) and Douglas' Lemma.

$i v) \Leftrightarrow v)$ : Consider the inner product and the associated norm on $\mathcal{F} \oplus \mathcal{H}$ as in (2.1). Then $G \in L(\mathcal{F}, \mathcal{H})$ is a $\left(\begin{array}{cc}I & 0 \\ 0 & T^{*} T\end{array}\right)$-optimal inverse of $V$ if and only if for every $f_{0} \in \mathcal{F},\left\|V G f_{0}-f_{0}\right\|^{2}+\left\|G f_{0}\right\|_{T^{*} T}^{2} \leq$ $\left\|V h-f_{0}\right\|^{2}+\|h\|_{T^{*} T}^{2}$ for every $h \in \mathcal{H}$ or, equivalently, $\left\|V G f_{0}-f_{0}\right\|^{2}+\left\|T G f_{0}\right\|^{2} \leq\left\|V h-f_{0}\right\|^{2}+\|T h\|^{2}$ for every $h \in \mathcal{H}$, that is, $G$ is a bounded global solution of (SMP).

ii $) \Leftrightarrow v i)$ : Suppose that $R(V)$ is closed, then $R\left(V^{*} V\right)=R\left(V^{*}\right)$. It can be seen that $R\left(V^{*}\right)=$ $R\left(V^{*} V\right) \subseteq R\left(T^{*} T+V^{*} V\right)$ if and only if $R\left(T^{*} T+V^{*} V\right)=R\left(T^{*} T\right)+R\left(V^{*} V\right)$. But this is equivalent to $\left(T^{*} T, N(V)\right)$ being compatible, see [10, Theorem 3.2].

In Theorem 4.6 it was proved that the existence of a bounded global solution of the classical smoothing problem (SMP) is equivalent to the existence of a $W$-optimal inverse for the weight $\left(\begin{array}{cc}I & 0 \\ 0 & T^{*} T\end{array}\right)$. In a similar way, in Theorem 3.2, the equivalence between the existence of bounded global solutions for WLSP and the existence of $W$-inverses was stated for a positive weight $W$. Motivated by this relation, in what follows we are interested in comparing $W$-inverses to $W$-optimal inverses. We begin with the following lemma.

Lemma 4.7. Let $W \in L(\mathcal{F} \oplus \mathcal{H})^{+}$with block form as in (4.9), $A \in L(\mathcal{H}, \mathcal{F})$ and $\hat{A} \in L(\mathcal{H}, \mathcal{F} \oplus \mathcal{H})$ be defined by $\hat{A} h=(A h, h)$. Then, there exists a $W$-inverse of $\hat{A}$ if and only if there exists a $W$-optimal inverse of $A$ and the equation

$$
\left(A^{*} W_{11} A+A^{*} W_{12}+W_{12}^{*} A+W_{22}\right) X=A^{*} W_{12}+W_{22}
$$

admits a solution. 
Proof. Suppose that $Z \in L(\mathcal{F} \oplus \mathcal{H}, \mathcal{H})$ is a $W$-inverse of $\hat{A}$, then $\hat{A}^{*} W \hat{A} Z(f, h)=\hat{A}^{*} W(f, h)$ for every $(f, h) \in \mathcal{F} \oplus \mathcal{H}$, i.e.,

$$
\left(A^{*} W_{11} A+A^{*} W_{12}+W_{12}^{*} A+W_{22}\right) Z(f, h)=A^{*} W_{11} f+A^{*} W_{12} h+W_{12}^{*} f+W_{22} h,
$$

for every $(f, h) \in \mathcal{F} \oplus \mathcal{H}$. In particular, if $h=0$, then

$$
\left(A^{*} W_{11} A+A^{*} W_{12}+W_{12}^{*} A+W_{22}\right) Z(f, 0)=A^{*} W_{11} f+W_{12}^{*} f, \text { for every } f \in \mathcal{F} .
$$

Therefore $Z_{1}(f):=Z(f, 0)$ is a $W$-optimal inverse of $A$. In the same way, if $f=0$, then

$$
\left(A^{*} W_{11} A+A^{*} W_{12}+A_{12}^{*} A+W_{22}\right) Z(0, h)=A^{*} W_{12} h+W_{22} h, \text { for every } h \in \mathcal{H} .
$$

Therefore $Z_{2}(h):=Z(0, h)$ is a solution of (4.11).

Conversely, suppose that $Z_{1} \in L(\mathcal{F}, \mathcal{H})$ is a $W$-optimal inverse of $A$ and $Z_{2} \in L(\mathcal{H})$ is a solution of (4.11). Let $Z: \mathcal{F} \oplus \mathcal{H} \rightarrow \mathcal{H}$ be defined by $Z(f, h):=Z_{1}(f)+Z_{2}(h)$. Then, clearly

$$
\hat{A}^{*} W \hat{A} Z=\hat{A}^{*} W .
$$

Also,

$$
\begin{aligned}
\|Z(f, h)\|^{2} & =\left\|Z_{1}(f)+Z_{2}(h)\right\|^{2} \leq\left(\left\|Z_{1}(f)\right\|+\left\|Z_{2}(h)\right\|\right)^{2} \leq\left(\left\|Z_{1}\right\|\|f\|+\left\|Z_{2}\right\|\|h\|\right)^{2} \\
& \leq\left(\max \left\{\left\|Z_{1}\right\|,\left\|Z_{2}\right\|\right\}\right)^{2}(\|f\|+\|h\|)^{2} \leq 2\left(\max \left\{\left\|Z_{1}\right\|,\left\|Z_{2}\right\|\right\}\right)^{2}\left(\|f\|^{2}+\|h\|^{2}\right) \\
& =2\left(\max \left\{\left\|Z_{1}\right\|,\left\|Z_{2}\right\|\right\}\right)^{2}\|(f, h)\|^{2} .
\end{aligned}
$$

Therefore $Z \in L(\mathcal{F} \oplus \mathcal{H}, \mathcal{H})$ and $Z$ is a $W$-inverse of $\hat{A}$.

The next proposition shows that certain optimal inverses can be seen as the weighted inverse of an associated operator.

Proposition 4.8. Let $A \in L(\mathcal{H}, \mathcal{F}), w \in L(\mathcal{H})^{+}$and $\hat{A} \in L(\mathcal{H}, \mathcal{F} \oplus \mathcal{H})$ be defined by $\hat{A} h=(A h, h)$. Then, there exists a $\left(\begin{array}{cc}I & 0 \\ 0 & w\end{array}\right)$-inverse of $\hat{A}$ if and only if there exists a $\left(\begin{array}{cc}I & 0 \\ 0 & w\end{array}\right)$-optimal inverse of A.

Proof. Suppose that there exists a $\left(\begin{array}{cc}I & 0 \\ 0 & w\end{array}\right)$-optimal inverse of $A$. By (4.10) and Douglas' Lemma, $R\left(A^{*}\right) \subseteq R\left(A^{*} A+w\right)$. This is $R\left(A^{*} A\right) \subseteq R\left(A^{*} A+w\right)$ and, this is equivalent to $R\left(A^{*} A\right)+R(w)=$ $R\left(A^{*} A+w\right)$. Hence, $R(w) \subseteq R\left(A^{*} A+w\right)$ and by Douglas's Lemma, the equation $\left(A^{*} A+w\right) X=w$ admits a solution. Therefore, by Lemma 4.7, $\hat{A}$ admits a $\left(\begin{array}{cc}I & 0 \\ 0 & w\end{array}\right)$-inverse. The converse follows by Lemma 4.7

\section{Acknowledgements}

We thank the anonymous referees for carefully reading our manuscript and helping us to improve this paper with several useful comments.

Maximiliano Contino was supported by CONICET PIP 0168. Guillermina Fongi was partially supported by ANPCyT PICT 2017-0883.

\section{References}

[1] Aiken J.G., Erdos J.A., Goldstein J.A., Unitary Approximation of positive operators, Illinois J. Math, 20 (1980), 61-72.

[2] Anderson W.N., Trapp G.E., Shorted Operators II, SIAM J. Appl. Math, 28 (1975), 60-71. 
[3] Antezana J., Corach G., Sampling theory, oblique projections and a question by Smale and Zhou, Appl. Comput. Harmon. Anal., 21 (2006), 245-253.

[4] Anselone P.M., Laurent P.J., A general method for the construction of interpolating or smoothing spline-functions, Numer. Math., 12 (1968), 66-82.

[5] Atteia M., Generalization de la définition et des propriétés des spline-fonctions, C.R. Acad. Sci. Paris, 260 (1965), 3550-3553.

[6] de Boor C., Convergence of abstract splines, J. Approx. Theory, 31 (1981), 80-89.

[7] Champion R., Lenard C.T., Mills T.M., An introduction to abstract splines, Math. Sci., 21 (1996), 8-16.

[8] Champion R., Lenard C.T., Mills T.M., A variational approach to splines, The ANZIAM J., 42 (2000), 119-135.

[9] Corach G., Fongi G., Maestripieri A., Weighted projections into closed subspaces, Studia Math., 216 (2013), 131-148.

[10] Corach G., Fongi G., Maestripieri A., Optimal inverses and abstract splines, Linear Algebra Appl., 496 (2016), 182-192.

[11] Contino M., Giribet J.I., Maestripieri A., Weighted Procrustes problems, J. Math. Anal. Appl., 445 (2017), 443-458.

[12] Corach G., Maestripieri A., Weighted generalized inverses, oblique projections and least-squares problems, Numer. Funct. Anal. Optim., 26 (2005), 659-673.

[13] Corach G., Maestripieri A., Stojanoff D., Oblique projections and Schur complements, Acta Sci. Math. (Szeged), 67 (2001), 337-256.

[14] Corach G., Maestripieri A., Stojanoff D., Oblique projections and abstract splines, J. Approx. Theory, 117 (2002), 189-206.

[15] Douglas R.G., On majorization, factorization and range inclusion of operators in Hilbert space, Proc. Amer. Math. Soc., 17 (1966), 413-416.

[16] Eldar Y., Sampling and reconstruction in arbitrary spaces and oblique dual frame vectors, J. Fourier Anal. Appl., 9 (2003), 77-96.

[17] Eldar Y., Werther T., General framework for consistent sampling in Hilbert spaces, Int. J. Wavelets Multiresolution Inf. Process., 3 (2005), 497-509.

[18] Fillmore P.A., Williams J.P., On operator ranges, Adv. Math., 7 (1971), 254-281.

[19] Holladay J.C., A smoothest curve approximation, Math. Tables Aids to Comp., 11 (1957), 233-243.

[20] Izumino S., Convergence of generalized inverses and spline projectors, J. Approx. Theory, 38 (1983), 269-278.

[21] Engl H.W., Nashed M.Z., New extremal characterizations of generalized inverses of linear operators, J. Math. Anal. Appl., 82 (1981), 566-586.

[22] Krein M.G., The theory of self-adjoint extensions of semibounded Hermitian operators and its applications, I. (Russian) Rec. Math. [Mat. Sbornik] N.S., 20(62) (1947), 431-495.

[23] Mitra S.K., Optimal inverse of a matrix, Sankhya Ser. A, 37 (1975), 550-563.

[24] Nashed M.Z., Inner, Outer and generalized inverses in Banach and Hilbert spaces, Numer. Funct. Anal. Optim., 9 (1987), 261-325. 
[25] Sard A., Approximation and variance, Trans. of the Amer. Math. Soc., 73 (1952), 428-446.

[26] Rao C., Mitra S.K., Theory and application of constrained inverse of matrices, SIAM J. Appl. Math., 24 (1973), 473-488.

[27] Ringrose J.R., Compact non-self-adjoint operators, Van Nostrand Reinhold Co., 1971.

[28] Schoenberg I.J., Contributions to the problem of approximation of equidistant data by analytic functions. Part A: On the problem of smoothing or graduation. A first class of analytic approximation formulae, Quart. Appl. Math., 4 (1946), 45-99.

[29] Schoenberg I.J., Contributions to the problem of approximation of equidistant data by analytic functions. Part B: On the problem of osculatory interpolation. A second class of analytic approximation formulae, Quart. Appl. Math., 4 (1946), 112-141.

[30] Shekhtman B., Unconditional convergence of abstract splines, J. Approx. Theory, 30 (1980), 237-246.

[31] Simon B., Trace Ideals and their applications, London Mathematical Society Lecture Note Series, vol. 35, Cambridge University Press, Cambridge, 1979.

[32] Smale S., Zhou D. X., Shannon sampling and function reconstruction from point values, Bull. Amer. Math. Soc., 41 (2004), 279-305.

[33] Weidmann J., Linear operators in Hilbert spaces, vol. 68., Springer Science \& Business Media, 2012. 